\title{
EL ESTADO COMO ALIADO. LOS MÉDICOS Y EL PROCESO DE ESTATALIZACIÓN DE LOS SERVICIOS SANITARIOS EN LA SEGUNDA REPÚBLICA ESPAÑOLA
}

\author{
Isabel Jiménez-Lucena \\ Ayudante de Facultad L.R.U. - Historia de la Ciencia - Facultad de Medicina. \\ Universidad de Málaga - Campus de Teatinos - 29080 Málaga (España).
}

\section{RESUMEN}

Las actitudes del colectivo médico ante la perspectiva de la intervención estatal en la organización y gestión de los servicios médico-sanitarios, y la dependencia de éstos del Poder público central, han marcado buena parte de las manifestaciones públicas de los profesionales de la medicina durante el siglo XX. En este trabajo se trata de analizar cuáles eran las expectativas de los médicos españoles respecto a la intervención del Estado en la actividad médico-sanitaria; y cuáles eran los elementos que constituían el modelo sanitario-asistencial propugnado por esta parte de los implicados en el sistema sanitario. Para alcanzar el objetivo propuesto se han analizado discursos de organizaciones corporativas y de la prensa profesional emitidos durante el periodo 1931-1936.

\section{SUMMARY}

The attitudes of the medical collective in the face of the perspective of the state intervention in the organization and administration of the medical-sanitarium services, and the dependenc of these on the public central Power, have marked an important part of the public statements of professionals of the medicine during the $\mathrm{XX}^{\text {th }}$ century. In this paper we tried to analyze which were the expectations of the Spanish physicians concerning the intervention of the State in the defended by the physicians implied in the sanitary system. In order to reach the proposed objective they have been analyzed speeches of corporate organizations and of the professional press emitted during the period (1931-1936). 
Como es sabido, los años Treinta fueron cruciales en la conformación de determinados aspectos característicos de las sociedades actuales ${ }^{1}$. En nuestro país este periodo histórico coincidió con la instauración de un régimen democrático que posibilitó la expresión de los distintos sectores sociales y, con ello, la agudización de la dinámica de interrelación entre grupos y clases, evidenciándose los conflictos y consensos existentes entre los mismos.

Por otra parte, las relaciones del Estado con las diversas posiciones de la estructura social es un tema sobre el que se han elaborado distintas teorías. Sin embargo, necesita de aportaciones empíricas que verifiquen el grado de validez de las mismas y muestren los intereses que los distintos grupos sociales tienen en la actuación del Estado.

En este trabajo se trata de analizar cuáles eran las expectativas de los médicos españoles respecto a la intervención del Estado en la actividad médico-sanitaria. Una cuestión que sigue estando llena de contradicciones que pueden ser clarificadas con el análisis histórico del problema ${ }^{2}$. Para alcanzar el objetivo propuesto se han analizado discursos de organizaciones corporativas como Colegios y otras asociaciones así como los de la prensa profesional. Como es sabido, estos foros pretenden ejercer poder e influencia en los procesos que tienden a señalar las líneas de desarrollo de los asuntos que afectan al colectivo organizado. El análisis de estos discursos se ha hecho teniendo en cuenta que éstos se construyen a partir de posiciones e intereses sociales determinados, a la vez que pretenden fundar y legitimar una realidad a la medida de los emisores ${ }^{3}$.

En este sentido partimos del reconocimiento de cierta heterogeneidad entre los profesionales de la medicina, tanto en la situación económica como aspectos ideológicos; pero, también consideramos que no se debía perder de vista el hecho de que los componentes de la categoría socioprofesional analizada formaban parte de un grupo social más amplio, una clase, que en términos generales se reconoce como burguesía ${ }^{4}$. Estas nociones nos fueron de gran ayuda en la comprensión y explica-

\footnotetext{
1 Véase a este respecto Ucelay-da Cal, E. y Veiga, F., El fin del segundo milenio (Un siglo de miedos apocalípticos, 1914-1989), en FONTANA, J. y UCELAY-DA CAL, E. (dirs.). (1994); Historia Universal Planeta, vol. 12, Editorial Planeta, Barcelona, pp. 135 y ss.

2 Recientemente, Rafael Huertas ha realizado un interesante análisis de aspectos relacionados con este tema en HUERTAS GARCÍA-ALEJO, R. (1995), Organización sanitaria y crisis social en España. La discusión sobre el modelo de servicios sanitarios públicos en el primer tercio del siglo XX, Fundación de Investigaciones Marxistas, Madrid.

3 Sobre estas propuestas de "enlazar construcción discursiva de lo social y construcción social de los discursos" puede verse CHARTIER, R. (1993), «De la historia social de la cultura a la historia cultural de lo social», Historia Social, n ${ }^{\circ}$ 17, 97-103, p. 103.

4 Acerca de la necesidad de insertar los grupos de presión, como el sector médico organizado, en el esquema global de las posiciones de clase y el lugar que aquél ocupa en las mismas, puede verse NAVARRO,
} 
ción de las opciones y propuestas que los componentes del colectivo médico elaboraron y defendieron respecto a la actuación del Estado en los servicios sanitarios. Así pues, tanto los intereses de grupo y de clase como las opciones ideológicas se mostraron útiles como clave interpretativa.

Las actitudes del colectivo médico ante la perspectiva de la intervención del Estado en la organización y gestión de los servicios médico-sanitarios, y la dependencia de éstos del Poder público central, han marcado buena parte de las manifestaciones públicas de los profesionales de la medicina durante el siglo XX. La expresión de opiniones respecto a esta cuestión no fue escasa durante el quinquenio republicano; debido, entre otras razones, a las expectativas de lo que se preveía como una inminente reforma sanitaria, producto de la necesidad de adaptación de estos servicios a la realidad socioeconómica de esta importante y crítica etapa histórica. El discurso de distintos miembros del colectivo médico, unas veces expresando opiniones a título individual, otras en representación de determinados sectores profesionales, constituye un cuerpo de propuestas a analizar, con objeto de poner de manifiesto cuáles eran los elementos que constituían el modelo sanitario-asistencial propugnado por esta parte de los implicados en el sistema sanitario.

\section{LA COMPARTIMENTACIÓN DE LA ACTIVIDAD MÉDICO-SANITARIA Y LA REDISTRI- BUCIÓN DE LOS RECURSOS}

Uno de los elementos clave en el discurso médico durante la Segunda República en relación a las propuestas de reforma sanitaria fue, sin duda, la reivindicación de la intervención del Estado en la realización de un proceso de redistribución de las inversiones públicas en actividades médico-sanitarias. El objetivo central era potenciar la financiación de determinados servicios de carácter preventivo, mientras otras parcelas de la actividad médica quedaban al margen. Incluso se proponía que los recursos provenientes de las partidas correspondientes a la asistencia benéfica, es decir de la medicina curativa individualizada gratuita para los más desfavorecidos, se fuesen trasvasando a los capítulos presupuestarios de los servicios de profilaxis. Es cierto

\footnotetext{
V. (1978), La Medicina bajo el capitalismo, Crítica, Barcelona, pp. 232-4 y 254-5. Por otra parte, existen numerosos estudios que sitúan a los componentes del colectivo médico entre las clases medias o burguesía; también se han realizado estudios empíricos a nivel local que muestran esta realidad, como las tesis de licenciatura acerca de los profesionales dirigidas por Juan Luis Carrillo y Jesús Castellanos: VILlarejo Álvarez, M. ${ }^{a}$ I. (1986), Profesionales sanitarios en la Málaga del último cuarto del siglo XIX, tesis de licenciatura inédita, Málaga; FERNÁNDEZ MARTíN, F. J. (1986), Profesionales sanitarios en la Málaga en el primer cuarto del siglo XX, tesis de licenciatura inédita, Málaga.
} 
que este tipo de actuaciones fomentarían una visión preventivista de la medicina ${ }^{5}$, pero también permitían reivindicar la protección, tanto social como económica, del Estado para labores que no podían ser ofertadas en el mercado; bien porque no tuviesen demanda, bien porque necesitaran de unos niveles de inversión no accesibles o difícilmente rentables para la actividad privada. Las actuaciones sanitarias, en su acepción clásica de lucha contra los morbos sociales, tenían estas características en, prácticamente, todas sus facetas. Necesidad de fuertes inversiones en infraestructura sanitaria (alcantarillado, abastecimiento de agua, etc.), de escasa o nula rentabilidad para la actividad privada, en tanto que las inversiones necesarias eran difícilmente capitalizables, al menos en porcentajes que procuraran beneficios "apetecibles"6, y una dudosa demanda en la parte correspondiente a la actividad médica preventiva ${ }^{7}$.

\subsection{Los discursos hegemónico y alternativo en la prensa profesional.}

En el sentido antes expuesto, en la publicación conservadora El Siglo Médico,$^{8}$ se sucedieron las declaraciones referentes a que la Sanidad era "la primera y principal obligación de cuantas deben ser atendidas y patrocinadas directamente por el Estado"; un Estado que tenía "el deber y la obligación de velar por la sanidad de la nación y la salud de sus súbditos, fuente y base del poderío de todo pueblo fuerte". Pero este deber lo habría de realizar con sumo cuidado para no perjudicar los intereses de la "clase médica"; pues modificar el ejercicio profesional en el ámbito de la

5 Sobre los intentos de coordinar aspectos asistenciales y preventivos de la actividad médica puede verse HUERTAS GARCíA-ALEJO, R.(1995), pp. 66-82.

6 Respecto a esta cuestión y el papel del Estado en la financiación de los servicios sanitarios puede verse: HEILBRONER, R. L. (1990), Naturaleza y lógica del capitalismo, Ediciones Península, Barcelona, pp. 88 y ss. y 144-7; NAVARRO, V. (1978), pp. 242-4, 264-6.

7 En este sentido podemos considerar las manifestaciones en torno a la inexistencia de un "ambiente sanitario" y la necesidad de fomentar el "espíritu higiénico" tan frecuentes en el discurso médicosanitario.

8 Como ha quedado señalado en ROdRígueZ OCAÑA, E.; Rosado CAMACHO, E.; MORENo RODRíGUEZ, R.M ${ }^{\mathrm{a}}$.(1985-86), «La polémica en torno a la ley de libertad de enseñanza en España, vista a través del periodismo médico (1861-1874)», Dynamis, 5-6, 245-57, El Siglo Médico era una publicación con larga tradición conservadora. Esta tradición la mantuvo en el periodo histórico aquí analizado, como hemos podido comprobar a través de los apoyos políticos que se ofrecían en sus páginas, vinculados siempre a las personalidades y grupos más conservadores del espectro político, como Albiñana de Renovación Española, o Bermejillo de la CEDA, por citar sólo dos ejemplos.

9 Entre las numerosas manifestaciones que se hicieron sobre este asunto pueden verse MARTíNEZ SALDISE, M. (1931), «Salus populi suprema lex est», El Siglo Médico, 88, nº 4069, 592; FERNÁNDEZ DE VELASCO, S. (1932a), «Mirando a la realidad», El Siglo Médico, 90, n⿳0十104, 155-6; y (1932b), «Propongo», El Siglo Médico, 90 n 4124, 687-8. 
llamada asistencia médica, entendida ésta como la actividad clínica individualizada, podría socavar los principios básicos de una "profesión liberal". Así, no debía exagerarse la intervención administrativa en la medicina y para ello, según el director de $E l$ Siglo Médico, Carlos $\mathrm{M}^{\mathrm{a}}$. Cortezo, había que definir y separar bien lo que era Sanidad y lo que era Asistencia Pública. ${ }^{10}$

La clave de una "buena" actuación pública estaba en la inversión de los recursos públicos en determinadas parcelas mientras otras debían permanecer totalmente al margen. Esto lo explicaba el colaborador de El Siglo Médico, Ramón Giner, utilizando lo que él mismo llamaba "el más clásico argumento contra el socialismo" que consideraba que el colectivismo estatal cegaba la fuente de eficacia que era dejar "manar el caudal de energía y de iniciativa de que es capaz cada hombre, acuciado individualmente por el afán de superarse y mejorar con respecto de sus convecinos". Y lo que era una regla general válida, todavía era más evidente en "la medicina social" entendida ésta como la asistencia de urgencia -es decir en afecciones agudasprestada a nivel domiciliario y privado. En cambio, para "enfermos crónicos, para cirugía, para la atención eficaz y más premiosa en dispensarios y laboratorios, todo cuanto desarrollen las corporaciones privadas o públicas nos parecerá poco"11. Es decir, el afán de superarse quedaría restringido a aquellas actividades que no supusieran una inversión importante para el médico, en cuanto a instalaciones o instrumental, e imposible de posponer para el paciente; mientras que para el ejercicio más complicado, en cuanto a la necesidad de una mayor inversión material o una menos apremiante necesidad de asistencia por parte del cliente, cabía la intervención del Estado, aunque el caudal de energía e iniciativa sufriera efectos similares a los de una sequía.

En definitiva, se trataba de mantener la diversidad de fuentes de beneficios y de impedir que se quebrara el "espíritu liberal" de la profesión. Para ello, se insistió en la diferenciación entre "lo sanitario" y "lo asistencial", y en la conveniencia de mantener la línea divisoria entre lo que era la asistencia a los "desheredados" y el resto de la actividad profesional clínica ${ }^{12}$. La ya clásica reivindicación de una rigurosa selección

10 Decio CaRlan [Cortezo, C.Ma.](1932a), «Lo mismo está que estaba», El Siglo Médico, 90, $\mathrm{n}^{\circ}$ 4101, 73-4; (1932b), «Un grito en la noche», El Siglo Médico, 90, nº 4114, 417-8.

11 GiNER, R. (1933), «Los igualatorios médicos contra el censo», El Siglo Médico, 92, nº 4153, 66-7.

12 Mantener separadas las actuaciones ejecutivas de los servicios de Sanidad y Beneficencia, era una forma eficaz de asegurar que la asistencia médica mantuviese una demanda privada, no mezclando una actividad a la que tenían derecho todos con otra en la que debía pasarse una rigurosa selección para formar parte del grupo beneficiario. Además, esta forma de prestación tenía la ventaja de poder ser fácilmente instrumentalizada, en caso de que conviniera desprestigiar las actuaciones médico-sanitarias pública, denunciando el abuso de estos servicios por parte de la población; George Rosen ha señalado la utilización de este argumento para destruir programas sanitarios públicos. ROSEN, G. (1985), De la 
de los integrantes de las listas de beneficencia pretendía lo que Gregorio Nieto, Secretario del Colegio de Médicos de Soria, expuso en los últimos meses de vida de la República, dándonos a conocer que el viejo problema no se había resuelto y continuaba en los mismos términos. En concreto, Nieto proponía, como solución a los problemas profesionales, que se delimitasen de forma precisa cuáles eran las funciones de la Sanidad y de la Beneficencia, es decir hasta dónde llegaría la intervención de los poderes públicos, con objeto de que se respetase escrupulosamente el ejercicio libre de la profesión, sin competencias "desleales". Los sanitarios funcionarios del Estado debían limitar su actuación a la vigilancia y prevención de la aparición de enfermedades epidémicas, así como los médicos de la Beneficencia a los enfermos pobres, desheredados, exclusivamente ${ }^{13}$. En ambos casos el Poder público aseguraría unos beneficios que nunca se obtendrían en el "libre mercado". Pero, exceptuando a los "pobres de solemnidad", que de ninguna manera podrían hacer frente a los gastos de asistencia médica por muy reducidos que éstos fuesen, el resto de la actividad asistencial debía someterse a los cánones del ejercicio liberal de la profesión.

Esta división ya había mostrado con anterioridad su utilidad en la construcción de un determinado discurso médico, pues había permitido que el director de El Siglo Médico, Francisco J. Cortezo, pudiese reivindicar "la organización de los servicios de Beneficencia y Sanidad de una vez para siempre y dependientes en absoluto del Estado" de forma que el inspector municipal de Sanidad y el médico de la Beneficencia pública sería un funcionario del Estado. Y, a la vez, mantener que "De modo paralelo a la función sanitaria del estado correrá el ejercicio profesional médico en todos sus aspectos, dentro de la más absoluta libertad e independencia, desde el ejercicio médico libre en el medio rural al de las capitales de mayor población. Para la organización del ejercicio médico se hace absolutamente preciso [...] la abolición, tajante, de todo servicio profesional médico prestado en compañías y sociedades y pagado en forma de sueldo global [porque] la socialización arbitraria de los servicios es la muerte del progreso científico y de la moral médica" ${ }^{14}$.

\footnotetext{
Policía Médica a la Medicina Social: ensayos sobre la historia de la atención a la salud, Siglo Veintiuno, México, pp. 373-4.

13 NiETO, G. (1936), «Sanidad, Beneficencia y ejercicio libre de la profesión», El Siglo Médico, 97, $\mathrm{n}^{\circ} 4283$, 48-9. Estos mismos argumentos habían sido expuestos por distintos autores a lo largo de los años que ocupó el régimen republicano. Véase, por ejemplo, ÁlVAREZ SIERRA, J.A. (1934a), «Hay que defender la Medicina rural», El Siglo Médico, 93, n $4195,503-504$.

14 DECIO CARLAN [Cortezo, F.J.](1935a), «De cómo varios diputados ruegan por escrito al Ministro de Trabajo», El Siglo Médico, 95, $\mathrm{n}^{\circ}$ 4237, 238-9. No deja de tener importancia que estas fuesen las bases del esquema programático que expuso F.J. Cortezo durante la campaña electoral de febrero de 1936, con el que pretendía guiar el voto de los profesionales hacia las fuerzas políticas conservadoras: DECIO CARLAN [Cortezo, F.J.](1936a), «El esquema de un programa sanitario y profesional médico», $E l$ Siglo Médico, 97, n 4283, 145-6 y (1936b), «Compás de espera», El Siglo Médico, 97, n 4283, p. 176.
} 
Así, los sectores más conservadores, en los que se ubicaba Francisco J. Cortezo, defendían como ideal un modelo bicompartimental donde no cabía la asistencia asegurada $^{15}$. Y para que este esquema funcionase había que asegurar lo que Cortezo llamaba "la severa aplicación de la función benéfica". Sólo los que demostraran encontrarse en la indigencia podrían acceder a una asistencia médica gratuita, siguiendo el modelo, propio del liberalismo, en el que sólo los "miserables", que no pudiesen resolver sus problemas en el mercado "libre", serían acogidos por el sistema asistencial público; marcándose, así, al beneficiado con el estigma de la incapacidad ${ }^{16}$.

En la publicación de corte reformista La Medicina Íbera ${ }^{17}$ se difundieron opciones que, salvando matices de gradación, perseguían los mismos fines que las propuestas en el semanario conservador antes analizado. Los argumentos en este caso consideraban que si se pretendía realizar una reforma sanitaria, en cierta forma ambiciosa, con una financiación insuficiente debía buscarse recursos ya existentes adaptándolos a las necesidades fijadas. En este sentido se defendía el traspaso de recursos desde los servicios benéficos, de asistencia médica individual, a la Sanidad; y el Estado, considerado como entidad "neutral", sin intereses que defender, era el más adecuado para realizar la reorganización de los servicios de forma "apropiada para la acción social a que le obliga el interés de la salud pública en lo que se refiere a la prevención de los contagios" ${ }^{18}$. De esta forma, si el Estado no podía hacer frente por si solo a esta labor, como aseguraba, entre otras autoridades sanitarias José Verdes Montenegro, debía intervenir para reordenar los recursos en favor del "bien común". En este caso los recursos de las "consultas públicas" debían contribuir al desarrollo de los servicios de carácter preventivo; es decir la Beneficencia podía y debía aportar recursos a la Sanidad ${ }^{19}$. Si tenemos en cuenta los beneficiarios de estas actividades, se estaba pidiendo una redistribución en la que perdían los más desfavorecidos en pro del "bien común".

15 La dinámica socioeconómica hizo que esta opción fuese, en su forma más radical, minoritaria, pues la asistencia asegurada fue, en general, aceptada; aunque con diversidad de modalidades.

16 El carácter punitivo del modelo de asistencialismo sólo para indigentes ha sido señalado en numerosas ocasiones; puede verse al respecto ARMAS, A. de (1993), «Concepto de salud y su evolución. El ser humano», en GONZÁLEZ DE CHAVES, $\mathrm{M}^{\mathrm{a}}$.A. (comp.), Cuerpo y subjetividad femenina. Salud y género, Siglo Veintiuno, Madrid, pp. 37 y ss.

17 La Medicina Íbera podemos situarla dentro de la corriente reformista. Su director, Fernando Coca, estuvo comprometido con Acción Republicana, y entre sus colaboradores habituales se encontraban hombres situados en los sectores más progresistas del espectro ideológico, desde Augusto Almarza al anarcosindicalista Polo y Fiayo.

18 VERDES MONTENEGRO, J. (1931), «Las consultas públicas de pecho y la acción sanitaria», La Medicina Íbera, 25, $\mathrm{n}^{\circ} 704$, CDXLIX.

19 VERDES MONTENEGRO, J. (1931), «De interés profesional. Reglamentación de las consultas», $E l$ Sol, 30 de julio. Verdes fue Director General de Sanidad entre septiembre y octubre de 1933. 


\section{ISABEL JIMÉNEZ-LUCENA}

También se expresó en La Medicina Íbera una tendencia aún mas próxima a las divulgadas en El Siglo Médico, que defendía con vehemencia un Estado con unos límites conceptuados en la tradición liberal. De esta forma, para José Álvarez Sierra, también colaborador de El Siglo, entre los peligros que acechaban al médico no era el menos dramático la socialización de la Medicina, pues "el comunismo amenazando a España, la socialización de la Medicina casi a las puertas de Europa; el seguro de enfermedad como máquina de asistir enfermedades defendido por algunos técnicos, se acercan tiempos en que dedicarse a la ciencia de Hipócrates tendrá graves dificultades"20. Dificultades que vendrían dadas, entre otras razones, por el hecho de que al convertir al médico en asalariado se le exigirían responsabilidades por su actuación técnica ${ }^{21}$. Así pues, el Estado debía tener una intervención mínima, en tanto que debía defender intereses colectivos pero nunca a costa de supuestos derechos individuales, aunque en realidad éstos fuesen intereses corporativos y de clase. En general, el límite del interés colectivo estaba en la posibilidad de negocio privado; si la actuación de los servicios públicos restaba posibilidades a la obtención privada de beneficios aquélla era rechazada. Las palabras de Álvarez Sierra son muy claras:

\footnotetext{
"la expansión poco meditada de los institutos sanitarios puede agravar la situación de la clase médica, ya que resulta frecuente el confundir la función de la Sanidad y profilaxis con la benéfica. Conocemos algunas capitales de provincia donde los Dispensarios antivenéreos, sólo han conseguido arruinar a los tres o cuatro especialistas que tenían consultorios particulares. En cambio, los beneficios de la higiene y de lucha contra la prostitución siguen casi igual. Los Dispensarios rurales de Higiene infantil, los antituberculosos y antipalúdicos, son un arma de dos filos, uno de los cuales se vuelve contra la actuación del médico libre, que es, en fin de cuentas, el que hace la verdadera Medicina."22
}

Otro ejemplo de exposición diáfana en el mismo sentido fue el del médico puericultor Rodríguez Pedreira, quien también consideraba que desde los centros sanitarios oficiales se hacía competencia desleal, pues la no delimitación estricta de la medicina preventiva y la medicina curativa daba como resultado que los médicos clínicos no enviaban a sus clientes a los Centros de Higiene, para hacer profilaxis, "ante el temor muy justificado de que sus ingresos sean menores al suponer que pueda entrar como médico en la familia, el que solamente debía dedicarse a la higiene preventiva". Por otra parte, el médico puericultor, o cualquier otro sanitario, bus-

20 ÁlVAREZ Sierra, J. (1932), «Responsabilidad», La Medicina Íbera, 26, n747, CLV.

21 Ibidem.

22 Álvarez SierRa, J. (1934b), «Problemas de la Medicina española. El malestar de la clase», La Medicina Íbera, 28, $\mathrm{n}^{\circ} 851$, CXCIII. Un texto de características similares a éste fue el publicado en $E l$ Siglo Médico, ÁlVAREZ SIERRA, J. (1934a). 
caría "otras actividades alejadas de su misión para atender a sus necesidades y a las de su familia" si no era bien retribuido 23 .

Ahora bien, aunque de forma menos extendida, frente a estas tendencias fuertemente compartimentadoras y diversificadoras se publicaron algunas propuestas que aun manteniendo un carácter más o menos diversificador y sin llegar a constituir apuestas claramente integralistas y colectivizadoras, sí suponían un paso adelante en este sentido; aunque lo que sobresalía en algunas de ellas era las ventajas que mostraban para determinados sectores de la profesión. Así, Francisco Bécares desarrolló un plan de funcionamiento de los servicios sanitarios basado en la integración de la actividad asistencial en los centros sanitarios que se habían puesto en marcha, siguiendo las recomendaciones de la Conferencia de Ginebra acerca de la Higiene rural. De esta forma, todo lo referente a sanidad y asistencia estaría organizado y dirigido por el Poder central pero financiado con la cooperación tanto individual como familiar, del Municipio, de la provincia y del Estado. El centro sanitario prestaría asistencia tanto a indigentes, por cuyos servicios pagarían los Ayuntamientos y Diputaciones, como a los trabajadores asegurados, servicios pagados por sociedades, mutualidades y cajas de seguros, y a los "pudientes", que pagarían privadamente al centro sanitario. Este sistema organizativo era definido por su autor como basado en un principio de socialización que garantizaría a todas las clases sociales, a todos los individuos, las prestaciones médico-sanitarias. Por tanto, tendría la ventaja de consagrar y hacer efectivo el derecho a la salud de todos los individuos. Pero, su alternativa de financiación, relacionada con las diferentes formas de acceso a los servicios sanitarios, marcaba el verdadero carácter diversificado del sistema propuesto y una protección absoluta de las remuneraciones del personal médico. En la financiación del sistema sanitario propuesto por Bécares los recursos aportados serían: Ayuntamientos, $5 \%$ presupuesto municipal +50 ptas por cada familia pobre; Particulares, 10 ptas por cada individuo de la familia; Diputaciones, $10 \%$ de su presupuesto; Estado, sostenimiento de los Centro y dotación de plazas de especialistas; Cajas del Seguro. El Estado, además, sería el organismo encargado de recaudar estos recursos y asegurar directamente las retribuciones a los profesionales de la medicina. ${ }^{24}$

Pero fue la opción propugnada por el médico anarcosindicalista Polo y Fiayo la que defendió, en medios profesionales, de forma más clara una medicina integral y colectivizada. Consideraba que la solución más acertada en cuanto a la eficacia de los servicios sanitarios era ir hacia una nacionalización o socialización conjunta de

23 Rodríguez PEDREIRA, J. (1935), «Puericultura - Pediatría. Instituto Provincial de Higiene Infantil», La Medicina Íbera, 29, $\mathrm{n}^{\circ}$ 897, XXXIX.

24 BÉCARES, F. (1933a), «Proyecto de organización de la asistencia médico-sanitaria rural», La Medicina Íbera, 27, $\mathrm{n}^{\circ}$ 832, CCLXXIII-IX y (1933b), «Más sobre nuestro proyecto de organización de la Asistencia médico-sanitaria rural. II» La Medicina Íbera, 27 (838) CDI. 


\section{ISABEL JIMÉNEZ-LUCENA}

asistencia y sanidad, ya que no se alcanzaría eficacia alguna si el Estado no coordinaba todos sus elementos clínicos, sanitarios y sociales ${ }^{25}$. Sin embargo, el propio autor de la propuesta reconocía lo improbable de su realización manteniendo que "La socialización, o mejor, la nacionalización de la Medicina en un amplio sentido social de derecho del pueblo, no es una forma asequible para esta República tímida y discreta que conserva con unción los últimos destellos de una burguesía moribunda; cuando la antorcha de la revolución haya quemado sus baluartes que todavía huelen a cirio y a incienso y haya destruido sus organizaciones heredadas de la vieja monarquía, entonces nacerá la sociedad que precisa la nacionalización de la Medicina"26. Aunque, por otra parte, veía ciertas expectativas de cambio basadas, no ya en el reconocimiento del derecho a la salud, sino en los intereses del colectivo médico y de la burguesía en general. En este sentido, aseguraba que "La medicina actual es esclava de las finanzas porque precisa grandes instalaciones, y como ello es inaccesible para el bolsillo del médico que consumió sus disponibilidades para serlo, preciso será, o que la ciencia sea una forma de explotación para enfermos y médicos, y los beneficios tan sólo para los capitalistas, como acontece en los tipos del seguro mercantil, o que se socialice". Además, la misma burguesía habría de considerar que aquella nacionalización sería "una forma más justa y completa, necesaria a la prosperidad del país"27.

\subsection{La expresión de lo hegemónico en organizaciones y reuniones médicas.}

Personajes que ocupaban cargos de representación en organizaciones profesionales como el entonces presidente del Consejo Nacional de los Colegios Médicos, Pérez Mateos, concretaba los límites de la intervención estatal, en los siguientes términos:

"Los centros secundarios deben multiplicarse, pero circunscribiendo sus funciones al asesoramiento, al diagnóstico, al auxilio del médico. Pero si derivan a la asistencia pública y son policlínicas subvencionadas por el Estado, resultarán perjudiciales para los médicos"28.

25 POLO y FIAYO, F. (1931a), «La Asamblea Antituberculosa Médico-Social», La Medicina Íbera, $25, \mathrm{n}^{\circ} 705$, CDLXIX.

26 POlO y FIAYO, F. (1931b), «¿Socialización o escarnio?», La Medicina Íbera, 25, $\mathrm{n}^{\circ}$ 727, CCCXX-II

27 POLO y FIAYO, F. (1931c), «Pesimismo profesional ante la posible implantación del Seguro de Enfermedad», La Medicina Íbera, 25, n 733, CDXXXI.

28 [PÉREZ MATEOS, J.] (1932), «Asamblea de Medicos Titulares Inspectores Municipales de Sanidad. La conferencia del Dr. Pérez Mateos», El Siglo Médico, 90 (4115) 446. 


\section{EL ESTADO COMO ALIADO. LOS MÉDICOS Y EL PROCESO DE ESTATALIZACIÓN DE...}

El peligro de esta clase de centros consistía en que eran de gran atracción por los medios materiales de que disponían y absorbían la clientela libre, "mermando los ingresos del rural y royendo su prestigio". El ejemplo más claro de que podían ser un elemento perturbador para los profesionales de la medicina lo constituía, según Pérez Mateos, el hecho de que las sociedades de seguros alemanas, cuando chocaban con sus médicos, les reducía a la obediencia amenazándoles con crear esta clase de centros. De esta manera, las declaraciones del máximo representante de la organización colegial nos daba a conocer la contradicción existente entre los intereses profesionales y la racionalidad científico-médica. A los médicos no les interesaba unos servicios públicos bien dotados y de acceso universal donde se practicase una medicina integral, individual y colectiva, curativa y preventiva, mientras que ésta era reconocida como la forma eficaz de hacer labor sanitaria ${ }^{29}$. Por el contrario, desde posiciones influyentes (prensa y cargos representativos) se construía, de forma mayoritaria, un discurso en el que el papel del Estado era mantener el fraccionamiento de la actividad médico-sanitaria, restringiendo las prestaciones de cada sector a determinados límites, considerado esto como la única forma de que "el beneficio que se haga al obrero no se saque de la carne del médico". ${ }^{30}$ Limitar los servicios terapéuticos, la actividad curativa individual, en definitiva "los servicios que busca la gente por su propia iniciativa", en los centros públicos, era una forma de procurar la pervivencia de una porción de ejercicio privado lo más amplia posible.

Ante la duda de que el Estado no cumpliera adecuadamente con este papel, se crearon organizaciones corporativas de tipo coyuntural, como la Unión de Defensa Médica (UDM), que pretendían asegurar la diversificación de la asistencia mediante la monopolización de la asistencia asegurada de la que los facultativos serían gestores y controladores, asegurando el mantenimiento del ejercicio libre para los enfermos pudientes y la beneficencia para enfermos pobres ${ }^{31}$. Esta misma posición había sido adoptada y dada a conocer tres años antes por el Sindicat de Metges de Catalunya. Esta organización mantenía que como medio de cumplir las orientaciones domi-

29 El propio Pérez Mateos había manifestado que la Sanidad en su concepto moderno comprendía tanto la higiene y medicina preventiva como la asistencia al enfermo, pues esta era considerada también labor profiláctica. Ibid., pp. 445-6. Por otra parte, Michael Davis en 1921 ya reconocía que el éxito de los centros sanitarios públicos estaba en la posibilidad de ofrecer asistencia tanto curativa como preventiva, individual como colectiva; véase ROSEN, G. (1985), pp. 376-7, la cita es de la página 377.

30 [PÉREZ MATEOS, J.] (1932), p. 446.

31 Esta concepción no sólo defendía una asistencia desigual para los enfermos sino que, de la misma forma, dejaba explícita una concepción jerárquica y elitista de la profesión definida por la visión negativa de la posibilidad de un trato de igualdad para todos los integrantes del colectivo médico en el caso de que la organización de la asistencia médica se desarrollase en función de opciones estatales. Respecto a todas las cuestiones relacionadas con la UDM véase MUT, J. (1934), «U.D.M. Cooperativa de Asistencia Médica», La Medicina Íbera, 28, nº 845, LIII-V. 
nantes en la Sociedad de Naciones referentes a Higiene y Sanidad, expuestas tras las deliberaciones de las reuniones de octubre de 1930 en Budapest, en diciembre del mismo año en París y en marzo de 1931 en Ginebra, se procediera a la reglamentación estricta del servicio igualado. Esta reglamentación, así como la organización de la asistencia médica en la región catalana, la elaboraría y controlaría el Sindicat. Las modificaciones que proponía respecto a la organización de la asistencia ya existente eran mínimas y referidas a un control en la remuneración y en el mercado de trabajo médico. La diversificación no era modificada y sólo los indigentes podrían acceder a un servicio médico gratuito a cargo del Municipio; el resto de la población abonaría una cuota en relación a sus medios económicos para tener asegurada la asistencia médica; esta cuota la recaudaría la Sección del Sindicat encargada de ello y no el profesional directamente. El Estado no tenía ninguna labor que desarrollar, la corporación se encargaría de todo ${ }^{32}$.

Por último, en dos importantes Congresos que se celebraron durante el periodo republicano para tratar los temas sanitarios: el I Congreso Nacional de Sanidad y el Primer Congreso Español Pro-Médico ${ }^{33}$, nos ha sido posible apreciar también la importancia que la redistribución de los recursos, en el sentido de que los servicios públicos se limitasen a parcelas no rentables del ejercicio profesional, y la compartimentación tenían en la noción que del intervencionismo estatal desarrollaron los profesionales de la medicina.

En este sentido, las ponencias que en ambos congresos defendió Enrique Bardají sobre la organización del Seguro Social de Enfermedad ${ }^{34}$ dejaban claro que el papel

32 (1931), Sindicato de Médicos de Cataluña. Asamblea ordinaria de Delegados del año 1931, La Medicina Íbera, 25, $\mathrm{n}^{\circ} 712$, XIV-XVI.

'33 El «Primer Congreso Español Pro Médico» se celebró en Zaragoza, los días 6-11 de octubre de 1935. El Primer Congreso Nacional de Sanidad se celebro en Madrid, los días 6-12 de mayo de 1934; sobre determinados aspectos del mismo puede verse RodríGUEZ OCAÑA, E.; MENÉNDEZ NAVARRO, A. (1988), «Objetivos y estructura profesional de la Medicina social en la II República. El Primer Congreso Nacional de Sanidad. Madrid. 1934», en Actas del VIII Congreso Nacional de Historia de la Medicina, vol. I, Departamento de Historia de la Medicina, Murcia, pp. 514-523.

34 El Inspector Provincial de Sanidad de Madrid, Enrique Bardají, que ocuparía la Subsecretaría de Sanidad y Asistencia Pública entre abril y mayo de 1935, fue el divulgador del Seguro de enfermedad en las reuniones profesionales. Fue el encargado de las ponencias referentes al tema en el Congreso Nacional de Sanidad de Madrid y del Congreso Pro Médico de Zaragoza. Respecto al texto de las ponencias puede verse R.H. DEL C. (1934), «El Primer Congresos Nacional de Sanidad», La Medicina Íbera, 28, $\mathrm{n}^{\circ}$ 862, CDLVII-CDLXIII. Esta ponencia fue publicada íntegramente por los Anales del INP de Mayo de 1934, pp. 641-9, dado su interés para la institución. BARDAJí, E. (1935), «Seguro Social de Enfermedad», La Medicina Íbera, 29, $\mathrm{n}^{\circ}$ 936, 489-94; y 29, $\mathrm{n}^{\circ}$ 938, CCCXIII. Bardají formaba parte de la subponencia oficial para el estudio de las relaciones del Seguro y la Sanidad junto con Aznar y Pittaluga, ambos integrantes de sectores políticos conservadores (católico y liberal, respectivamente); la afinidad de Bardají con ambos nos la muestra su afirmación en el Congreso de Madrid respecto a que era necesario "ir adentrando la medicina social en el pueblo por medio del espíritu cristiano". El Sol, 8 mayo 1934. 
que el Estado debía desempeñar era el de redistribuidor de los recursos ya existentes, obligando a invertir en materia sanitaria preventiva parte de los medios económicos de los servicios asistenciales de la Beneficencia y del Seguro Oficial. Además, el Estado se ocuparía de realizar las inversiones iniciales de establecimiento de las instituciones sanitarias, limitándose después al sostenimiento de los Institutos de Higiene y los Centros rurales de Sanidad y Medicina Preventiva; dejando la administración de los servicios de asistencia asegurada a otras instancias.

Pero fue, sin duda, Santiago Ruesta, miembro de la Sanidad Nacional y Subsecretario de Sanidad y Beneficencia entre enero y febrero de 1936, quien, sobre todo en la ponencia que defendió en Zaragoza denominada "Política Sanitaria. Sanidad Nacional.", expuso con detalle un amplio programa en el que se trataban los distintos aspectos del ejercicio médico. Así, se establecía una especie de clasificación en la que la Medicina preventiva se identificaba con la Medicina del Estado; la asistencia social con la Medicina "de los pobres" y la Medicina privada, se hacía corresponder con la Medicina "de los ricos". Sobre todas ellas debía ejercer el Estado su influencia, pero de muy distinta manera. La Medicina preventiva (vacunaciones, luchas contra las llamadas enfermedades evitables como las antipalúdicas, antivenéreas, etc.) eran actividades propias del Estado, y debían ser llevadas a cabo en su totalidad por el mismo, a través de sus organizaciones sanitarias de carácter técnico; las razones de ello eran:

\begin{abstract}
"en primer lugar, la escasa cultura de la población, que no sabe todavía valorar la aplicación de una vacuna o el examen médico periódico de un sujeto sano y que, por tanto, obliga a ciertos métodos coactivos, que sólo al Estado es lícito emplear; segundo, la organización profesional médica en casi todo el mundo, con exclusión del médico rural español, está basada fundamentalmente en el ejercicio libre del arte de curar. La terapéutica es casi el único recurso económico del médico libre [luego] parece difícil interesar al médico liberal en la disminución de la morbilidad; tercero, los actos llevados a cabo por la Medicina preventiva no pueden, en ningún modo, ser caprichosos, sino fruto de estudios [requiriendo] conocimientos especiales que no tienen por qué ser exigidos al profesional libre; y cuarto, la ejecución de la Medicina preventiva por cuenta del Estado apenas perjudica a la profesión libre, pues si bien es cierto que existen enfermedades, como el paludismo y la sífilis, cuya profilaxis consiste en la esterilización de portadores, no es menos cierto que al Estado le interesa fundamentalmente el tratamiento social de estos enfermos, y no consideramos difícil el poder establecer de modo exclusivo este tratamiento (blanqueo de la sífilis) si encontramos las garantías suficientes para que una vez hecho inofensivo el enfermo, pueda ser entregado a la asistencia social, si se tratase de un pobre, o a la Medicina privada si se tratase de un rico"35.
\end{abstract}

35 RUESTA, S. (1936), «Primer Congreso español Pro-Médico. Política Sanitaria», La Medicina Íbe$r a, 30, \mathrm{n}^{\circ} 948,57-63$. Una propuesta similar fue la elaborada y publicada con anterioridad por $\mathrm{F}$. BÉCARES; véase nota 24 de este trabajo. 


\section{ISABEL JIMÉNEZ-LUCENA}

Todas las funciones de la Medicina preventiva serían realizadas en Centros sanitarios - Centros secundarios y primarios de Higiene rural y Centros terciarios o Institutos provinciales de higiene-, por médicos funcionarios del Estado bien remunerados para prohibirles toda actividad profesional ajena a la profilaxis pública o privada.

La parte médica de la asistencia social sería responsabilidad, hasta que los seguros sociales se hiciesen cargo de la misma, de las instancias locales, quienes, vigiladas y controladas por el Estado y junto con la iniciativa privada, fomentada y encauzada por el Estado, serían las que debían ocuparse de este tipo de asistencia, entre otras razones, por cuestiones económicas.

Por otra parte, los servicios médicos de carácter curativo e individual para la población no indigente entrarían en el ámbito de la Medicina privada. Bajo ningún concepto debía considerarse estas actividades como función del Estado, ni a los que la realizaran, incluso en la población indigente, funcionarios del Estado. Así pues, en los Centros de Higiene no debía prestarse estos servicios pues en caso contrario se cometería una grave infracción contra la seriedad que debía imperar en todos los actos del Estado, inmiscuyéndose en un campo de actividades que no le pertenecía. Como ejemplo de las consecuencias de esta injerencia expuso que la misma había sido causa de serias dificultades en el desarrollo de la organización de Centros de Higiene. En este mismo sentido, el colectivo profesional no debía pedir que los titulares pasasen a formar parte del Cuerpo de Sanidad Nacional, sino reclamar que la incorporación, en todo caso, se hiciese atendiendo al desempeño de ciertos actos administrativos de carácter público, reservándose el libre ejercicio de la profesión clínica, pues lo contrario sería "vender a bajo precio el carácter liberal de nuestra profesión". Y, lo que era peor, si se aceptaba que la asistencia médica domiciliaria a los pobres debía prestarse por funcionarios a sueldo, se incapacitaba a los profesionales para defenderse de las empresas que pretendiesen "poner a sueldo a los médicos que presten asistencia a sus asociados casi pobres". El problema de la creciente necesidad de una cierta tecnificación para el ejercicio clínico de la Medicina lo resolvía Ruesta fácilmente: la Administración sanitaria podría autorizar a los médicos el ejercicio privado en las instalaciones del Estado, en los Centros de Higiene equipados con el material necesario. De esta forma se aseguraría que todos los núcleos de población contasen con asistencia médica, facilitando a los facultativos las condiciones del desempeño de su trabajo ${ }^{36}$.

Ahora bien, como muestra la propia intervención de Ruesta, lo conflictivo del modelo propuesto se hacía manifiesto en el nivel asistencial más básico: el de los médicos titulares de los núcleos rurales, pues estos profesionales venían pidiendo desde décadas atrás que se reconociese su labor asistencial como parte de la respon-

36 Ibidem. 


\section{EL ESTADO COMO ALIADO. LOS MÉDICOS Y EL PROCESO DE ESTATALIZACIÓN DE...}

sabilidad del Estado en materia sanitaria, produciéndose un conflicto entre los intereses de un grupo en su totalidad y los de un sector de dicho grupo. Y en el Congreso Pro Médico se pidió la incorporación a la Sanidad Nacional por el doble concepto de su actividad: sanitaria y médica; no sólo el servicio en las cuestiones de medicina preventiva debía depender del Estado ${ }^{37}$. Esta situación llevó a que se intentara una solución de excepcionalidad que, sin pretenderlo, abría una vía a la intervención estatal en los servicios de medicina curativa de forma generalizada. Entre las conclusiones y acuerdos adoptados en este tema de la "Política Sanitaria. Sanidad Nacional. Sanidad rural" se aprobó que la administración de la salud pública debía hacerse a cargo de funcionarios del Estado debidamente especializados y de forma incompatible con otra actividad profesional, pero se excluía de esta norma a los médicos titulares que prestaran servicios en los centros primarios; en todos los partidos médicos habría un centro primario dirigido por el médico o los médicos, en caso de existir un solo centro y varios médicos, de Sanidad y Asistencia pública. Estas medidas debían realizarse de forma completa en un plazo de cinco años ${ }^{38}$.

Sin embargo, el equilibrio de fuerzas tras la victoria del Frente Popular debió hacer más creíbles las advertencias de Ruesta respecto a las "peligrosas consecuencias" de "vender a bajo precio el carácter liberal" de la profesión, y los titulares acabaron aceptando que el Estado les retribuyera sólo en concepto de inspección sanitaria. En fechas muy próximas al inicio de la guerra civil, las peticiones del Comité ejecutivo de la Asociación Oficial de Médicos de APD (antiguos médicos titulares inspectores municipales de Sanidad) al Subsecretario de Sanidad y Beneficencia y al Presidente de la República, en relación a la intervención del Estado, se centraron en cuestiones relativas a los intereses económicos del colectivo. No había ninguna propuesta de reforma sanitaria ni de modificación del ejercicio profesional; sólo se pedía que el Estado se encargase de recaudar las retribuciones de los titulares, y aportara la tercera parte de las mismas, estimada en unos diez millones de pesetas, para aliviar las cargas de los Ayuntamientos, en concepto de la labor de inspección. A cambio, se ofrecía el esfuerzo cotidiano y la separación de los extremismos, saturando la labor médica de "serenidad y esperanza", virtudes éstas de la profesión médica ${ }^{39}$.

37 (1936), Primer Congreso Español Pro Médico. Ponencias», La Medicina Íbera, 30, $\mathrm{n}^{\circ}$ 948, pp. 63-6. En la ponencia sobre Sanidad rural del Congreso, a cargo de Antonio Ossorio Bolaños, Joaquín Ruiz Heras y Nicolás Martín Cirajas, todos ellos destacados miembros de la organización médico-titular, el eje del contenido del discurso estuvo basado en la necesidad de incorporación del médico titular a la Sanidad Nacional.

38 (1935), «Conclusiones definitivas y acuerdos del Primer Congreso Español Pro Médico, recientemente celebrado en Zaragoza», La Medicina Íbera, 29, $\mathrm{n}^{\circ}$ 939, CCCXXXVII.

39 Asociación Oficial de Médicos de A.P.D.(1936a), «Sigue en pie el grave problema de los débitos de los Ayuntamientos a los médicos titulares», La Medicina Íbera, 30 (968) CCCLXXXIX; y (1936b), El Siglo Médico, 97, $\mathrm{n}^{\circ}$ 4296, 383-4. 
Ya en los últimos momentos de la República en paz, la posición de los organismos colegiales no había variado, básicamente, respecto al mantenimiento y la creación de compartimentos estancos dentro del sistema sanitario. Ante la información pública abierta por el Gobierno referida al proyecto de unificación de los Seguros Sociales, la respuesta que emitió el Colegio de Médicos de Madrid contenía, en el apartado que trataba sobre las prestaciones, las siguientes sugerencias: "El Seguro debe bastarse a sí mismo y con sus propios medios para el cumplimiento de todas sus obligaciones. [...] no deben establecerse coordinaciones ni prestaciones de las Beneficencias al Seguro, con el doble daño de desvirtuar la finalidad específica de las Beneficencias, ya insuficientes para atender a sus usuarios legales, los indigentes, o inferir el agravio que significa dar servicios gratuitos a quien cotiza para recibirlos"; además tampoco debía involucrar en sus servicios a la Sanidad pública, "cuya alta misión estatal nada ni nadie debe desviar"40.

En definitiva, la opinión hegemónica en medios profesionales era que la actividad médico-sanitaria debía quedar perfectamente compartimentada, con objeto de que no se produjesen injerencias desde la actividad pública en las parcelas consideradas de ejercicio privado. Así, por muy artificial que fuese establecer líneas divisorias entre las actividades médico-sanitarias, era imprescindible para mantener la liberalidad de la profesión, ${ }^{41}$ en una situación de clara tendencia a la salarización del ejercicio médico, ${ }^{42}$ y legitimar la ambivalencia profesional que permitía mantener las ventajas de ser funcionario y profesional liberal al mismo tiempo, evitando los inconvenientes de ambas situaciones.

\section{FOMENTO Y PROTECCIÓN DEL CORPORATIVISMO MÉDICO}

Durante la Segunda República, la corriente corporativa se expresó de forma particularmente manifiesta en el seno del colectivo médico, aunque el auge del corporativismo frente al liberalismo estricto se hizo evidente ya en la década de los veinte, como ha señalado Rafael Huertas. ${ }^{43} \mathrm{El}$ discurso médico se estaba adaptando a una realidad cambiante, a la vez que procuraba influir en la dirección que tomasen los

\footnotetext{
40 Recogido del texto reproducido en Colegio Oficial de Médicos de Madrid (1936), «Comparecencia del Colegio de Médicos de Madrid en la información pública sobre el Seguro Social de Enfermedad», El Siglo Médico, 97, n 4306, 655-8.

${ }^{41}$ Esta diferenciación se ha planteado y difundido desde unas premisas liberales y burguesas, como se ha explicitado en MAESTRO, A. y HUERTAS, R. (1992), La salud y el Estado: los servicios sanitarios públicos entre el bienestar y la crisis, Fundación de Investigaciones Marxistas, Madrid, pp. 101-2.

42 VILlaCORTA BAÑOS, F. (1989), Profesionales y burócratas: Estado y poder corporativo en la España del Siglo XX, 1890-1923, Siglo Veintiuno, Madrid.

43 Huertas García-Alejo, R. (1995), p. 131.
} 


\section{EL ESTADO COMO ALIADO. LOS MÉDICOS Y EL PROCESO DE ESTATALIZACIÓN DE...}

posibles cambios en el ámbito de la Medicina. La posición del colectivo médico, en tanto que segmento de la burguesía, estaba modificándose ante un movimiento obrero que iba adquiriendo una relevante posición; esta coyuntura estaba provocando que la idea del intervencionismo estatal, entendido éste en sentido liberal, estuviese sumando adeptos, que veían en la extensión del campo intervenido grandes ventajas. ${ }^{44}$

En estas circunstancias, la necesidad de contar con una fuerza homogénea ${ }^{45}$ se planteaba como una cuestión básica, debido a que había que ser capaz de conseguir que la actuación del Poder público garantizara los intereses de los profesionales, en la nueva forma de asistencia médica que se preveía como necesaria ante la evolución del sistema económico y social ${ }^{46}$. La garantía mencionada significaba el control

44 En nuestro país, con la implantación de la II República, el Partido Socialista había ocupado determinadas cotas de Poder y el sindicalismo de clase estaba en auge. En muchas ocasiones, esto asustaba a determinados estratos de la clase media, que consideraban que las clases subordinadas se beneficiarían a costa de ellos; para evitarlo buscaban, con especial insistencia, según señala VILlACORTA BAÑos, $F$. (1989), pp. 487 y ss., una entidad protectora que supiera neutralizar las posiciones "aventajadas" de los más desfavorecidos. En este sentido, se pedía al Estado que asegurara a los médicos sus retribuciones y sus estatus; al considerarlo desde la perspectiva liberal una instancia "neutral", no importaba quiénes gobernaran para reivindicar su actuación. Uno de los miembros de la familia Cortezo, Víctor $\mathrm{M}^{\mathrm{a}}$, que llegó a ocupar el cargo de Inspector de Instituciones Sanitarias, fue muy explícito respecto a este asunto. En su discurso de recepción como académico de la Academia Nacional de Medicina, mantenía que el Estado debía ocuparse de dar protagonismo a la iniciativa privada aunque, al mismo tiempo, debía cuidar de los límites de la misma respecto a que la iniciativa privada "no fuera de productores por lo propensos que eran éstos al monopolio". Cortezo, V.Mª.(1935), «El momento sanitario. Parte doctrinal del discurso leído por el Excmo. Sr. Dr. Víctor María Cortezo en el acto de su recepción como académico de la Nacional de Medicina el 29 de abril de 1935», El Siglo Médico, 95, n 4247, 530-39.

45 Para conseguir homogeneidad en la acción del colectivo y con ello constituirse en una fuerza capaz de ejercer una presión suficiente para lograr determinados privilegios, era necesario lograr una pacificación interna en el colectivo médico. Para ello, se pedía que el Estado interviniese como organismo pacificador de los conflictos existentes dentro del colectivo, posibilitando la unificación de los intereses de los distintos sectores profesionales. A este respecto puede verse: PITTALUGA, G. (1935), «Contestación del académico de número Prof. Gustavo Pittaluga [al discurso de recepción de V.M ${ }^{\mathrm{a}}$. Cortezo]», El Siglo Médico, 95, $\mathrm{n}^{\circ}$ 4247, 539-41; Álvarez SIERRA, J. (1933), pp. 583-4; DECIO CARLAN [Cortezo, F.J.] (1935b), «La Asamblea de Granada», El Siglo Médico, 96, n 4256, 17-8; JiMÉNEZ CANO, F. (1936), «Los médicos rurales y la República», La Medicina Íbera, 30, n 974, XIX-XX; GARCÍA AYUSO, J. DE Dios (1933a), «El poder público y la profesión médica», La Medicina Íbera, 27, $\mathrm{n}^{\circ}$ 799, CLV; y (1933b), «La crisis médica y su patogenia. Remedios», La Medicina Íbera, 27, $\mathrm{n}^{\circ}$ 810, CCCLXIII; (1934), «Solemne Asamblea de Colegios Médicos. Se ratifica la unión de la clase», El Siglo Médico, 93, nº 4216, 359-62; (1934), «Información de la Asamblea de Juntas de Colegios Médicos. Ponencias interesantes», El Siglo Médico, 93, $\mathrm{n}^{\circ}$ 4217, 389-91; (1934), «Conclusiones aprobadas por la X Asamblea de Juntas de Gobierno de los Colegios Médicos españoles celebrada en San Sebastián», El Siglo Médico, 93, n 4219, 451-2.

46 El cambio no se veía sólo como necesario, sino, también ineludible. El presidente del Colegio, Dacio Crespo Álvarez, proponía el estudio de una alternativa que estableciera "nuevos cauces que den efectividad a una socialización de la Medicina, forzosa e indispensable, pero sin quebrantos o con los 


\section{ISABEL JIMÉNEZ-LUCENA}

exclusivo de la actividad médico-sanitaria por parte de los profesionales de la medicina, con el objetivo primordial de impedir cualquier interferencia en la libertad de actuación que, de forma unilateral, se adjudicaban como derecho irrenunciable los profesionales de la medicina; además de asegurar un mínimo, al menos, de ingresos económicos a buena parte del colectivo.

Así, cuando C.M $\mathrm{M}^{\mathrm{a}}$. Cortezo aseguraba que la función sanitaria era la que mayor independencia de acción necesitaba y, por ello, debía depender exclusivamente del Estado, estaba reivindicando el control de la actividad médico-sanitaria, a través de un Estado corporativo que eliminara cualquier otra fuente de intervención diferente a la profesional. Para cumplir esta misión la institución estatal era considerada superior a cualquier otra, dado "el prestigio impositivo de la totalidad autoritaria del Estado" 47 . Era, pues, en el sentido de facilitar la formación de una corporación médica investida de autoridad suficiente para hacer cumplir preceptos, en el que Carlos $\mathrm{M}^{\mathrm{a}}$ Cortezo reivindicaba un Estado interventor.

menos quebrantos posibles para nosotros los médicos", pues oponerse a cualquier cambio era "ir, por adelantado, al fracaso", dado que las fuerzas sociales contemporáneas, tanto socialistas como demócratacristianas y católicas, reivindicarían un cambio como lo habían hecho en otros países. CRESPO ÁLVAREZ, D. (1931a), «Ante el Seguro Social de Enfermedad», El Siglo Médico, 88, n' 4051, 110-2. Profesionales afines al ideario republicano liberal, como era el caso de uno de los fundadores de la Agrupación al Servicio de la República, Gregorio Marañón, veían en la actuación del Estado una vía de solución de problemas estructurales del sistema socioeconómico. Para Marañón era evidente que en un plazo breve la Medicina se socializaría "en toda la tierra"; la causa sería la crisis económica que provocaría la conversión de la Medicina en una función del Estado, en la cual "el enfermo del tipo medio tendría derecho a ser asistido por el Poder público y los médicos encargados de este servicio tendrían una remuneración asignada, un sueldo, y nada más". Pero no había que preocuparse por este último aspecto porque siempre habría mecanismos "para recompensar a los mejor dotados de inteligencia, voluntad o ambición frente a los perezosos o los tontos". MARAÑón, G.(1931), «Consideraciones acerca de la Medicina contemporánea» La Medicina Íbera, 25, $\mathrm{n}^{\circ}$ 724, CCLVII-IX. En la misma corriente de opinión se situaban otros colaboradores de La Medicina Íbera. Para F. de Bergós Ribalta era de una "evidencia aplastante" que la Medicina caminaba a ser una función del Estado; la causa de ello era la evolución económica de los pueblos. La "penuria mundial", llevaría, ineludiblemente, por la ruta de una socialización completa de la Medicina; el que esta reforma la hiciese el Estado sería una garantía para los intereses de los médicos, que no existiría en el caso de ser llevada a cabo por entidades no estatales. BERGÓs RIBALTA, F. de (1934), «Al servicio de los intereses profesionales», La Medicina Íbera, 28, $\mathrm{n}^{\circ}$ 850, CLXXI-III.

47 Decio Carlan [Cortezo, C.M ${ }^{\mathrm{a}}$.](1931a), «Por fin o por principio», El Siglo Médico, 88, nº 4060, 337; y (1931b), «En el Parlamento», El Siglo Médico, 88, n 4063, 421-2. Carlos M ${ }^{\mathrm{a}}$ Cortezo era un ejemplo de "primate médico" y prototipo de hombre del antiguo régimen restauracionista en el que había tenido una activa vida política. Como buen conservador, pensaba que el Estado debía asegurar el "bien general", entendido éste como el mantenimiento del orden social vigente; clásicamente estas nociones iban unidas a las actuaciones sanitarias relacionadas con las enfermedades infecto-contagiosas, consideradas como problemas de orden público y, por tanto, justificantes de actividades autoritarias, para las que sólo estaban legitimados los agentes del Estado, pues, como es sabido, éste monopoliza la fuerza impositiva en las sociedades modernas. 


\section{EL ESTADO COMO ALIADO. LOS MÉDICOS Y EL PROCESO DE ESTATALIZACIÓN DE...}

Para los profesionales liberales conservadores, en general, la intervención del Estado debía limitarse a la ordenación del sector, de forma que los intereses de la corporación médica quedasen a salvo. Gustavo Pittaluga, diputado de la derecha liberal en el primer bienio republicano y director de la Escuela Nacional de Sanidad, sostenía que era necesaria la plena intervención del Poder público en el ajuste y coordinación de la vasta labor sanitaria que había que realizar. El Estado debía poner en manos de los técnicos la dirección de estos servicios y solucionar los problemas de tipo profesional; una vez realizada esta labor no tenía por qué continuar interviniendo ${ }^{48}$.

En el mismo sentido, Víctor $\mathrm{M}^{\mathrm{a}}$. Cortezo aseguraba que era un ideal antiguo de los sanitarios el "ver llegar hasta el distrito la influencia de la organización central"; sin embargo, criticaba "la política de absorción por el Estado de todas las actividades sanitarias, practicada durante los primeros años de República" (la Lucha Antituberculosa, la Liga contra el Cáncer), considerando que había sido muy gravoso para la economía del Estado y había anulado la iniciativa privada. Así que, aunque se había desarrollado de forma rápida la organización sanitaria durante el periodo republicano, el mal funcionamiento se debía a que los técnicos habían perdido protagonismo frente a la dependencia y orientaciones políticas de los Gobiernos. La solución que proponía, como buen liberal, era librar al Estado de la carga que suponía el monopolio de las actividades sanitarias, tanto preventivas como curativas, devolviendo a la iniciativa privada el protagonismo que tenía antes de la instauración de la República (Patronatos, Ligas) e incluso ampliándolo. Sin embargo, esta propuesta no equivalía a la liberalización del "mercado sanitario", pues éste debía tener unos límites que Cortezo señalaba claramente: la iniciativa privada podía ser "de tipo religioso, filantrópico, patronal o en forma de cooperativa siempre que no fuera de productores por lo propensos que eran éstos al monopolio"49.

La conveniencia de estos tipos de límites estaban implícitas en propuestas como que el Estado se encargara de recaudar las retribuciones de los médicos titulares; se "sacrificaba" así el "espíritu liberal" del contrato directo paciente-médico a cambio de asegurarse el cobro de los servicios. ${ }^{50}$ Pero, no sólo esto sino que, con estas peti-

48 PITtaluga, G. (1935), pp. 539-41. Este argumento sobre la función que debía cumplir un organismo estatal, como un Ministerio de Sanidad, había sido expuesto ya en otras ocasiones; uno de sus defensores era Álvarez Sierra; véase, por ejemplo, ÁlVAREZ SIERRA, J. (1933), 583-4.

49 CORTEZO, V.M ${ }^{\mathrm{a}}$. (1935), 530-39.

50 Se trataba de que alguna entidad con autoridad, preferentemente el Estado, se hiciese cargo del cobro de los haberes, para que el médico no tuviese que intervenir en ello. Se llegó a proponer que el Estado cobrase a través de los impuestos de contribución la "derrama necesaria" para abonar directamente a los titulares por concepto de titular e igualas. Véanse: FERNÁNDEZ DE VELASCO, S. (1932c), «¿Sería una solución?», El Siglo Médico, 89, n 4082, 260-1; (1932a), pp. 155-6; (1932b), pp. 687-8; y (1933), «El problema médico rural radica en los mismos médicos», El Siglo Médico, 92, n 4170, 529; 


\section{ISABEL JIMÉNEZ-LUCENA}

ciones se estaba intentando, también, como explicitó el colaborador de El Siglo Médico Anastasio Pérez González, que, al no existir una relación contractual directa, el cliente, el enfermo, no exigiera al médico ${ }^{51}$. En este sentido, las palabras de Sisinio Crespo son paradigmáticas; para este profesional la situación ideal del titular era que tuviese un sueldo como funcionario del Estado, por los servicios de tipo sanitario que prestara, y, a la vez, fuesen médicos del seguro de enfermedad cuando éste se implantase, percibiendo otra remuneración de una "Sociedad solvente"; de esta for-

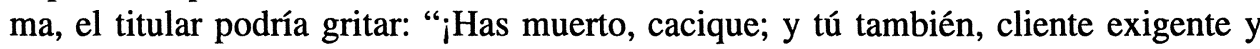
moroso!" 52 . Se pidió, como señalaba las palabras de Sisinio Crespo, que el Estado liberara a los sanitarios del "cacicato de los municipios". Pero los caciques frente a los que se pedía protección eran, según los rumores de pasillo que recogió El Siglo Médico durante la Asamblea de Médicos Titulares de 1932 , los Ayuntamientos republicanos ${ }^{53}$, los que, según el conservador Albiñana, "aún tienen el cinismo de predicar reivindicaciones sociales y la emancipación proletaria" 54 . Y es que, desde determinadas posiciones, se vio en la intervención del Estado una forma de evitar el control directo de la actividad profesional por parte de los usuarios, esgrimiendo una actitud caciquil de los Ayuntamientos y los "pueblos" respecto a los profesionales 55 . El Estado debía procurar que los médicos titulares-inspectores municipales de Sanidad no tuviesen más dependencia que la necesaria de las autoridades sanitarias superiores, iniciando un proceso conducente a la constitución de una tecnocracia ${ }^{56}$.

PÉREZ GonZÁlez, A. (1935), «Medios de dotar decorosamente a los Médicos titulares», El Siglo Médico, 95, $\mathrm{n}^{\circ}$ 4230, 21-3; ARRANZ, S. (1935), «Sugerencias de la Asamblea de las clases sanitarias de Segovia», El Siglo Médico, 95, n 4240, 327; DomínguEZ, A. F. (1935a), «Hasta aquí se llegó» El Siglo Médico, 95, $\mathrm{n}^{\circ}$ 4238, 268-9 y (1935b), «Colaboremos», El Siglo Médico, 95, n 4253, 688-90; COMITÉ Central de Enlace de las Clases Sanitarias (1935), El Siglo Médico, 96, $\mathrm{n}^{\circ} 4260,176$; PAADÍN EsTRELlA, R. (1936), El Siglo Médico, 97, n 4288, 176.

51 PÉrez GONZÁlez, A. (1935), 21-3.

52 CRESPO, S. (1936), El Siglo Médico, 97, nº 4287, 151-2. Sisinio Crespo era médico titular y colaborador habitual de El Siglo Médico.

53 En los comentarios que El Siglo Médico publicó de las sesiones de la Asamblea general de la Asociación, el redactor hace referencia a que en los pasillos se comentaban los atropellos de los Municipios del nuevo régimen y se defendía la actuación de la Dictadura que mejoró la situación de la clase. $\mathrm{L}$. $\mathrm{R}$. (1932), «La Asamblea general del Cuerpo de Médicos Titulares», El Siglo Médico, 89, nº 4075, 67-72.

54 (1935), «Emancipación de la clase médica rural (I): El Dr. Albiñana nos habla de los palpitantes problemas de la Sanidad española», El Siglo Médico, 95, n 4242, 410-2.

55 CRESPO, S. (1932), «En tanto en cuanto llega la ley sobre el Seguro de Enfermedad», El Siglo Médico, $90, \mathrm{n}^{\circ} 4125,716-8$.

56 Como es bien sabido, la tecnocracia es un sistema político basado en la preeminencia de los especialistas y su influencia determinante en la decisión política, y de alguna manera excluyente de los no expertos en la participación de los asuntos públicos. El término tecnocracia se empezó a utilizar en 1931 por Howard Scott. Véase CHORDÁ, F.; MARTíN, T.; RIVERO, Y. (1990), Diccionario de términos históricos $y$ afines, $3^{\mathrm{a}}$ ed., Ediciones Istmo, Madrid, p. 304. 


\section{EL ESTADO COMO ALIADO. LOS MÉDICOS Y EL PROCESO DE ESTATALIZACIÓN DE...}

Todos estos aspectos fueron tratados de forma sistemática en el ya mencionado proyecto de funcionamiento de los servicios sanitarios que elaboró y publicó Francisco Bécares. Las prestaciones médico-sanitarias serían tanto de financiación pública como privada, pero esta última no seguiría el modelo clásico liberal sino que se haría por concertación bien de unidades familiares o sociales (sociedades, mutualidades de asistencia, incluso las Cajas de seguros sociales) con los Centros sanitarios municipales y comarcales y no con los profesionales directamente; el personal cobraría del Estado y no de las entidades particulares o colectivas. Este sistema organizativo "pondría a cubierto al médico de toda asechanza contra su independencia funcional y ayudaría a conjurar en gran parte la crisis económica que atraviesa la profesión". Bécares trataba de mostrar las ventajas que su propuesta tendría para el colectivo médico-titular, integrándolos en los centros sanitarios, dependientes de la Sanidad provincial, desapareciendo la Beneficencia municipal y resolviéndose así los problemas económicos y de dependencia local de estos profesionales; cuestiones éstas que no se solucionarían sin que se realizase un cierto grado de nacionalización de servicios asistenciales y sanitarios ${ }^{57}$.

Profesionales de reconocidas tendencias socialista, como Augusto Almarza, se manifestaron en el mismo sentido. Aseguraba Almarza que los médicos titulares afines al Partido Socialista venían defendiendo la socialización de la Medicina "como ideal a conseguir" desde antes de la dictadura. Con la República se había extendido un ambiente favorable a la socialización de la Medicina fuera de la clase. Este cambio había que considerarlo fundamental, pues, según Almarza, llevaba implícito que si el Estado no realizaba la socialización de la medicina la haría la sociedad, prescindiendo del Estado y de los médicos o contra los médicos; prueba de ello era que en las poblaciones se iban multiplicando las Sociedades benéficas, mutualistas, cooperativas, etc., que prestaban asistencia médico-farmacéutica ${ }^{58}$. El modelo de "socialización" propugnado por Almarza, basado en una nacionalización de la actividad médica ${ }^{59}$, partía de la necesidad de resolver los problemas profesionales del colectivo médico-titular y vincular a los profesionales la dirección de las entidades administrativas; por tanto, concebía al Estado, básicamente, como una entidad protectora de los intereses corporativos. ${ }^{60}$

57 BÉCARES, F. (1933a), pp. CCLXXIII-IX; y (1933b), p. CDI.

58 AlmarZA, A. (1931a), «Notas breves», La Medicina Íbera, 25, nº 725, CCLXXXII; y.(1931b), «Comentarios de actualidad», La Medicina Íbera, 25, $\mathrm{n}^{\circ} 732$, CDXIII.

59 Las primeras manifestaciones de Almarza acerca de este tema, durante los primeros años del siglo XX, han sido recogido en VILlACORTA BAÑOS, F. (1989), pp. 487 y ss. En HUERTAS GARCÍA-AlEJO, R. (1995), pp. 132 y ss., se analiza el discurso de este médico en los primeros años del siglo.

60 AlmarZA, A. (1934), «El año que acaba y el año que empieza», La Medicina Íbera, 28, $\mathrm{n}^{\circ} 843$, IX; (1935), «Comentarios al Reglamento. La Medicina Íbera, 29, n 901, CIX-XI; (1935), «El ejercicio de la Medicina ante la evolución del Derecho. IV», La Medicina Íbera, 29, n 920, CDLXXIX-XIII; 
Desde esta perspectiva no era extraño que, como el mismo Almarza aseguraba, en aquellos momentos los profesionales sanitarios aparecieran "apoyando decididamente, enérgicamente si se quiere, una ley y unas disposiciones ministeriales que acentúan considerablemente la tendencia intervencionista del Estado", aunque, como él mismo manifestaba, no siempre hubiese sido así; pues, en otros momentos, "la clase" había aplaudido también la tendencia contraria, por falta de conocimiento de la evolución de la conciencia jurídica contemporánea que tendía al colectivismo ${ }^{61}$.

En este sentido, las organizaciones corporativas habían tratado el asunto del papel que debía desempeñar el Estado, en cuanto al desarrollo y funcionamiento de los distintos servicios médico-sanitarios, desde los primeros momentos del régimen republicano; incitadas, principalmente, por los preceptos que se propusieron en el proceso de discusión del texto constitucional, referentes a los servicios sanitarios tanto preventivos como curativos (Sanidad, Beneficencia y Seguros Sociales). El Colegio de médicos de Zamora fue rápido en su reacción, con el propósito de que fuesen los profesionales de la medicina los que elaboraran las pautas de organización y funcionamiento de los servicios sanitarios, de forma que quedasen a salvo los intereses del colectivo. Entre otras cosas, había que conseguir que la Sanidad fuese función del Estado para "defender a los titulares de la ruina social, económica y espiritual", ya que consideraba que las Cajas Aseguradoras controladas por el proletariado y los administradores explotarían a los profesionales. La Junta directiva del Colegio expuso el marco reivindicativo que los profesionales debían reclamar para defender sus intereses. La demanda clave, considerada como "el primer capítulo" del "credo" médico, quedaba sintetizada en la ya vieja frase "la Sanidad debe ser función exclusiva y preferente del Estado". Esta cuestión, que venía defendiéndose desde 1916, ahora se veía como una solución ante el problema de la implantación del Seguro Social de Enfermedad; había que lograr "redimir a la SANIDAD de la tutela tantas veces impertinente de las Corporaciones municipales" y protegerla "aún más de la esclavitud y de la tiranía a que pueden condenarla definitivamente las CAJAS ASEGURADORAS". El Estado debía intervenir en este asunto, pues se consideraba que la Sanidad estaba por encima de cualquier ideal político. Por ello, los colegiados de Zamora, en palabras de su presidente D. Crespo, no obstaculizarían jamás las mejoras que redundasen en beneficio del proletariado español, pero éste debía quedar al

(1935), «Los subdelegados de Medicina», La Medicina Íbera, 29, n 941, CCCLIII; (1935), «Las restricciones y la actualidad profesional», La Medicina Íbera, 29, n 935, CCLI-V; (1936), «Ordenación sanitaria», La Medicina Íbera, 30, n 961, CCLXV-VII.

61 Almarza, A. (1935), «El ejercicio de la Medicina ante la evolución del Derecho. V» La Medicina Íbera, 29, $\mathrm{n}^{\circ}$ 921, III. 


\section{EL ESTADO COMO ALIADO. LOS MÉDICOS Y EL PROCESO DE ESTATALIZACIÓN DE...}

margen del control y administración de los servicios sanitarios, siendo los técnicos, a través del Estado, los únicos cualificados para ello. ${ }^{62}$

La intención de que los servicios médicos quedasen controlados directamente por el Estado, sin intermediaciones que pudieran resultar desventajosas para los profesionales, fue expuesta también por otros organismos corporativos. En la Asociación de Médicos Titulares pedían que el Estado actuara como protector frente a las entidades no profesionales, como eran los responsables de los gobiernos locales, desde agencias controladas por profesionales como la Dirección General de Sanidad, a través de los inspectores provinciales de Sanidad, que debían ser los únicos que intervinieran en el nombramiento y separación de los facultativos titulares ${ }^{63}$. Este sector pedía al Estado protección incluso de otros sectores de la profesión; así, los Poderes públicos debían ocuparse también de que cuando se instituyese el Seguro Social de Enfermedad fuesen estos funcionarios, que se llamarían médicos de Sanidad y Asistencia Pública, los que prestasen con preferencia sus servicios médicos y no se constituyera un Cuerpo médico propio e independiente del Cuerpo de Asistencia Pública ${ }^{64}$.

Las peticiones del Comité Nacional de Defensa de las Clases Sanitarias, formado por miembros directivos de todas las organizaciones oficiales de sanitarios, fueron en la misma línea: conseguir que se les asegurara a los sanitarios la remuneración por los servicios contratados con los Ayuntamientos y la independencia respecto a las autoridades no profesionales, pidiendo que todas las decisiones respecto a los profesionales y la profesión dependieran de técnicos, es decir de las autoridades sanitarias ${ }^{65}$.

De la misma manera, en el foro del Primer Congreso Español Pro-Médico, se propuso que en el Consejo Nacional de Sanidad no hubiese representación alguna que no fuese la del Estado, entendida como la representada por la más alta jerarquía sanitaria nacional; sólo sanitarios, nada de representaciones de otros colectivos que

62 Crespo Álvarez, D. (1931a), pp 110-2; (1931b), «El Colegio de Médicos de Zamora contesta al Dr. Saldise», El Siglo Médico, $88, \mathrm{n}^{\circ} 4052,141-2$.

63 (1931), El Siglo Médico, 88, nº 4069, 593-4; L. R.(1932), pp 67-72.

64 (1936), Primer Congreso Español Pro Médico. Ponencias», pp. 63-6. No fue ésta la primera ni la última vez que desde un sector del colectivo se pedía la protección del Estado frente a otros sectores de la profesión. Véase: GARCíA AYUSO, J. DE D. (1933a), p. CLV; y (1933b), p. CCCLXIII; JIMÉNEZ CANO, F. (1936), pp. XIX-XX.

65 En este sentido, el Comité de Vizcaya publicó sus propuestas, entre las que destacaba, para su estudio en los distintos Colegios Oficiales, pedir que la D.G.S. o la Subsecretaría estuviese ocupada por un técnico y no sujeta a los vaivenes de la política y dentro de la D.G.S. se constituyeran las Secciones de Medicina, Farmacia, Veterinaria, Odontología, Practicantes y Matronas, y al frente de cada Sección estuviese un titulado de cada una de dichas profesiones nombrado por la clase a la que representara. Véase (1935), «Actuaciones de los Comités provinciales de Defensa de Clases Sanitarias», La Medicina Íbera, 29, n 901, CXXVI; (1935), «Comité de Defensa de las clases sanitarias», La Medicina Íbera, 29, $\mathrm{n}^{\circ} 904$, CLXXVI. 


\section{ISABEL JIMÉNEZ-LUCENA}

sólo servirían "para dificultar y entorpecer las orientaciones sanitarias". 66 Por otra parte, se aseguraba que la tutela y el control de los servicios sanitarios y asistenciales por parte del Estado favorecía los intereses de los profesionales ${ }^{67}$.

Pocas palabras hemos encontrado, en el marco de las fuentes analizadas, frente a este exceso de corporativismo que llevaba a propuestas tecnocráticas. Entre ellas las de Pando Baura; quien, si bien propugnaba el fortalecimiento de la autoridad del médico, criticaba al colectivo profesional precisamente el hecho de que siempre hubiese pedido al Estado medidas que protegieran intereses corporativos, pero nunca se había preocupado de elaborar un programa eficiente de lo que debía ser la Sanidad oficial. Además, entendía Pando que el control de la actividad médica no se debía sustraer de sus más directos beneficiarios; por tanto, no debía funcionarizarse, por ejemplo, la actividad de los médicos titulares que debían "ser nombrados por Ayuntamientos y Juntas de vecinos". ${ }^{68}$ Por su parte el anarcosindicalista Polo y Fiayo veía en los sindicatos la instancia apropiada para regular y encauzar todas las cuestiones relacionadas con el mantenimiento y restablecimiento de la salud; aunque, en última instancia, serían las manos de los médicos las únicas capaces de darles la orientación más conveniente a la asistencia médica y aspectos sanitarios. Sin embargo, esto sólo sería posible con una transformación estructural de la sociedad en su conjunto en favor de los más desfavorecidos, y no con la constitución de un poder tecnocrático apoyado en el Estado ${ }^{69}$.

66 PalanCA, A. (1935), «Ponencia sobre Sanidad Nacional», El Siglo Médico, 96, n 4270, 447-52.

67 BARDAJí, E. (1935), pp 489-94; Palanca, A.(1935).

68 PANDO BAURA, J.L. (1931), «El problema de los titulares. Hay que dar más medios de trabajo al médico», La Medicina Íbera, 25, $\mathrm{n}^{\circ}$ 703, CDXIX; (1933), «Nuestra Sanidad Oficial», La Medicina Íbera, 27, $\mathrm{n}^{\circ} 827$, CLXXIX. Para analizar las propuestas de este sanitario hay que tener en cuenta que era el promotor de una entidad de carácter particular como fue el Instituto Nacional de Sanidad y Pedagogía, que pretendía fuese acogida por la Dirección General de Sanidad.

69 POLO y FIAYO, F. (1931d), «Sindicato Único de Sanidad. Sección de Médico», La Medicina Íbera, 25, nº 726, CCCIV; y (1931b), p. CCCXXII. 